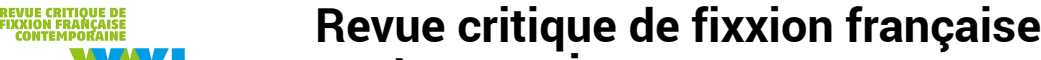 \\ XXI contemporaine

\section{Écritures en lutte dans le cyberespace}

Existe-t-il une littérature hacktiviste?

\section{Aurélien Maignant}

\section{OpenEdition}

Journals

Édition électronique

URL : https://journals.openedition.org/fixxion/532

DOI : $10.4000 /$ fixxion.532

ISSN : 2295-9106

Éditeur

Ghent University

Référence électronique

Aurélien Maignant, «Écritures en lutte dans le cyberespace », Revue critique de fixxion française contemporaine [En ligne], 20 | 2020, mis en ligne le 15 juin 2020, consulté le 17 mai 2022. URL : http:// journals.openedition.org/fixxion/532; DOI : https://doi.org/10.4000/fixxion.532

Ce document a été généré automatiquement le 17 mai 2022

\section{cc) (†) $\odot$}

Les contenus de la Revue critique de fixxion française contemporaine sont mis à disposition selon les termes de la licence Creative Commons Attribution - Pas d'Utilisation Commerciale - Pas de Modification 4.0 International. 


\section{Écritures en lutte dans le cyberespace}

Existe-t-il une littérature hacktiviste?

\section{Aurélien Maignant}

1 L'heure n'est plus aux constats timides sur l'importance croissante d'internet pour l'exercice du pouvoir. La métaphore spatiale devenue courante d'un huitième continent n'est pas anodine : les états et les $\mathrm{GAFAM}^{1}$ se réunissent aujourd'hui lors de sommets spécialisés comme l'E-G8 ${ }^{2}$ pour se répartir le cyberespace, comme autrefois les puissances coloniales attablées autour d'une carte se partageaient le monde. La numérisation croissante de nos existences, de nos manières de communiquer, de nos transactions marchandes ou même de nos dispositifs créatifs, engendre des mutations du contrôle ${ }^{3}$. "La cybernétique s'est historiquement trouvée devant de multiples critiques, aussi bien sur le terrain des sciences que sur celui des implications sociales et politiques de la logique du contrôle dont elle est à la fois une manifestation et une source fondamentale" mais plus personne ne peut nier que "l'ouverture d'un champ techno-organique continu est au fondement de notre expérience actuelle du monde"4. Alors la théorie politique s'adapte, invente de nouveaux logiciels critiques (comme la déconstruction du capitalisme computationnel ${ }^{5}$ ) ou identifie des zones à défendre encore peu explorées (comme nos attentions ${ }^{6}$ ). Alors les résistances s'adaptent, les militantismes changent, découvrent de nouvelles praxis (comme l'ensemble des hacktivismes qu'on abordera ici), mettent en place des sittings digitaux, détournent les nouveaux médias en espaces d'auto-organisation ou en journaux de combat ${ }^{7}$.

2 Ces militantismes numériques montrent qu'une partie de celles et ceux qui disposent d'une maitrise technique du cyberespace entend bien y exporter les combats du réelespace ${ }^{8}$, parce que "ça commence à se voir [...] que certains types de logiciels sont de l'autre côté de la barricade"'. À vrai dire, la brève mais déjà riche histoire du web suffit à montrer que l'utopie d'une neutralité technique ne s'est jamais concrétisée. Les récits de la première génération de hackeureuse's montrent que les laboratoires du M.I.T. (où apparait l'internet contemporain) étaient idéologiquement divisés entre d'une part celles et ceux qui voyaient les langages de programmation comme les racines d'un 
réseau utopique permettant l'accessibilité et la gratuité de la connaissance à l'échelle mondiale et d'autre part l'intérêt de certain'e's praticien'ne's ${ }^{10}$, des entreprises et des états pour la privatisation et la marchandisation des réseaux. Les hackeur-euse-s originelle's comme Richard Stallman racontent aujourd'hui un combat lentement perdu contre la dépossession de leurs outils, et comment cette "capture"11 progressive a forgé le world wide web ${ }^{12}$. Ces cyber-anarchistes et les nombreuses variantes de leurs idéologies sont aujourd'hui minoritaires, mais encore très actif.ve's dans les communautés Open, comme Linux, où se diffusent des systèmes d'exploitation et des logiciels dont le code source est accessible et modifiable par chaque utilisateur.trice ${ }^{13}$.

Aujourd'hui, le terme de hack désigne essentiellement la manipulation des échanges ou des communications par la réécriture du code ou le détournement de langages de programmation, un geste pouvant être mis au service d'intérêts gouvernementaux, personnels ou constituer un mode de résistance, une praxis militante à laquelle on réserve le terme d'hacktivisme. Pour ses principauxale's théoricien'ne's, l'hacktivisme apparait comme une pratique désobéissante adaptée aux mutations actuelles de la domination, ce qui repose sur l'hypothèse que nous vivons un moment historique lors duquel le contrôle peut être fragilisé par de simples gestes de transformation du réseau. Le principe d'un détournement localisé des infrastructures à des fins contestataires - type d'action distinct de la propagande ou du sabotage par exemple n'est évidemment pas nouveau, il faut plutôt constater que le terme de hack sert aujourd'hui à en proposer des systématisations. On a ainsi pu observer l'apparition de pratiques résistantes non-digitales comme le riot hacking ${ }^{14}$, le gender hacking ${ }^{15}$ ou le urban hacking ${ }^{16}$ ainsi que la multiplication de travaux de recherche en théorie des arts : étudier comment des installations "hackent l'esthétique" ${ }^{17}$, délimiter les frontières du hackart vidéo (datamoshing, databending, etc.) ${ }^{18}$ et des pratiques natives au web ( $h$ tml-art, ASCII art, etc. ${ }^{19}$ ), comprendre les liens entre hack et peinture ${ }^{20}$, voire même repenser les avant-gardes littéraires du XXe siècle comme des gestes relevant du hack ${ }^{21}$. Pour autant, on trouve peu d'études sur les dispositifs qui se présentent simultanément comme hacktivisme et comme littérature, du moins comme objets esthétiques digitaux conférant une fonction politique à leur textualité. On voudra essayer de circonscrire ici certaines conditions de possibilité d'une écriture hacktiviste (éventuellement de dispositifs hacktivistes littérarisants ${ }^{22}$ ) qui peut devenir une praxis à l'intérieur du berceau conceptuel du hack: le web. On reviendra sur certains aspects des luttes du cyberespace, en théorie comme en pratique, tout en abordant différents exemples qui en exploitent les possibilités et répondent à des questions inattendues : qu'est-ce qu'un générateur algorithmique de propagande ? Le texte de Farenheit 451 peut-il brouiller les protocoles de surveillance de la NSA ? Peut-on pirater les pages web des banques pour les remplacer par de la poésie?

\section{Langages naturels : Agit-Prop 2.0}

4 On peut constater d'emblée que l'apparition des littératures numériques offre aux écritures militantes plusieurs axes de réinvention formelle. Le plus évident ne tient pas à la littérarité en elle-même, mais à la capacité du net à multiplier les énergies: amplifier la visibilité des messages et diversifier leurs publics. Celles et ceux qu'on appelle simplement "activistes numériques" rappellent qu'il n'est pas nécessaire de savoir coder pour faire d'internet un outil politique. Plusieurs des hacktivistes les plus 
importante's de ces dernières années considèrent même qu'aujourd'hui la manipulation des technologies est devenue secondaire et appellent "troisième génération" du hack les démarches qui se contentent d'utiliser le pouvoir de démultiplication de l'information en alimentant des médias alternatifs ${ }^{23}$, en transformant les réseaux sociaux en espaces d'auto-organisation spontanés ou en réinventant le tractage dans des mail-lists pirates ${ }^{24}$. Dans cette perspective, on envisage aisément le huitième continent comme un lieu de réplication des rapports de forces réels et les réseaux comme des rues dans lesquelles il faut réapprendre à faire de la propagande, à une échelle qui devient rapidement planétaire.

5 Cette puissance de multiplication du web a engendré par exemple des forums d'écriture anarchistes qui, dans des dizaines de langues, ouvrent de nouvelles possibilités d'organisation et de création, ainsi que la numérisation et la mise en ligne gratuite d'essais classiques de la théorie militante sur des bases de données libres ${ }^{25}$. On constate aussi que certaines avant-gardes politiques contemporaines écrivent directement sur la toile, ce qui a une influence immédiate sur la manière dont elles conçoivent leurs textes. C'est le cas par exemple du manifeste xénoféministe dont les chapitres et les fragments ont d'abord été conçus pour s'adapter au scroll (défilement) des pages web contemporaine ${ }^{26}$, avant d'avoir été réaménagés pour leur publication traditionnelle. Il faudrait sans doute évoquer encore l'immense champ de la fanfiction, réécriture libre et collective des fictions transmédiales dominantes, qui s'inscrit fréquemment dans une dynamique de lutte (renversement des rapports de pouvoir productifs et auctoriaux, réécritures antisexistes/antiracistes, etc.) et fait partie des exemples invoqués par les rares travaux qui se préoccupent des croisements entre hack et littérature ${ }^{27}$.

Outre la multiplication des énergies, on songe aussi à une évolution formelle importante: le passage de la linéarité de l'imprimé à des dispositifs interactifs. Plusieurs études avancent aujourd'hui l'idée que les écritures numériques disposeraient d'une performativité particulière, liée au fait que les dispositifs textuels réagissent aux actions des lecteur.trice $\mathrm{s}^{28}$. Dans une perspective politique, cette performativité permet de réinventer la fonction agonistique et/ou mobilisatrice de l'écriture militante: l'interactivité intrinsèque $\mathrm{du}$ web rendrait la textualité plus directement injonctive, plus apte à susciter l'action. On peut penser à un exemple devenu canonique de la littérature numérique francophone intitulé Agir ${ }^{29}$, un "récit interactif" conçu par Serge Bouchardon et une équipe de développeur-euse's dans l'intention assumée d'explorer ce nouvel ordre performatif. À travers différents tableaux quotidiens, l'application pour smartphone propose de suivre le récit d'un entretien d'embauche échoué, ironisant sur certains poncifs de l'injonction libérale à la productivité et à la réussite par le travail ("Prouvez-moi que vous saurez être dynamique [...] Refusé. Un échec de plus à oublier"), dans un dispositif à la première personne et sans personnage délimité pour favoriser l'identification de tous.tes. Après l'expérience oppressive de l'entretien échoué, l'utilisateur.trice est invitée à agiter violemment son téléphone pour recomposer une phrase. La secousse agit sur la textualité, qu'un algorithme recompose, et dont le résultat affiche généralement une incitation à passer à l'action (par exemple “je veux l'anéantir") : l'interactivité des dispositifs programmés explore la possibilité d'une nouvelle co-présence et d'une co-modification lecture-texte.

7 Il est difficile de considérer Agir comme un projet proprement militant et, pour le moment, les tentatives francophones qui entendent conférer une radicalité aux littératures numériques semblent rares, mais quelques brèches apparaissent. C'est le 
cas d'une jeune maison d'édition francophone fondée en 2018 en Suisse alémanique : les éditions Abrüpt. Cette "fabrique d'antilivres" ${ }^{30}$ s'inscrit dans la longue tradition de l'édition de combat anarchiste dont elle reprend un ensemble de codes et de pratiques en les adaptant au monde numérique. Outre un catalogue protéiforme qui affiche des fictions, des essais politiques et quantité d'hybrides, Abrüpt met à disposition des "micro-antilivres" gratuits, des textes radicaux, conçus pour pouvoir être rapidement imprimés sur une imprimante personnelle ou de bureau, pliés et agrafés en brochures aisées à répandre. Ici, la mise à disposition d'un PDF préformaté entend faciliter et rénover la diffusion de fanzines, de tracts ou de manifestes artistiques. Se revendiquant du hack en théorie comme en pratique (mais invoquant aussi les corpus anarchistes et situationnistes des $\mathrm{XIX}^{\mathrm{e}}$ et $\mathrm{XX}^{\mathrm{e}}$ siècles), nombre de ces antilivres sont pensés pour s'insinuer dans les configurations médiales contemporaines : les modes de publication innovants d'Abrüpt entendent s'inscrire directement dans les rapports médiarchiques ${ }^{31}$. Un des exemples les plus parlants est le versionnage en bots, comptes automatisés qui publient à intervalles réguliers des extraits générés aléatoirement de textes propagandistes sur les réseaux sociaux (Facebook, Twitter, Instagram, Mastodon, etc.). Encore embryonnaire, le principe envisage un mode de publication qui mime et détourne la saturation attentionnelle organisée par les réseaux sociaux dominants, un tractage géré par des programmes autonomes qui cherchent à surcharger les canaux de diffusion. Constituant un dispositif majeur de captation attentionnelle, les murs (walls) d'accueil ${ }^{32}$ sont immédiatement apparentés aux murs des villes, et rendus susceptibles, comme leurs homologues non-virtuels, de devenir la cible de campagnes d'affichage. Abrüpt explore aussi des formats connus des littératures numériques non-militantes, comme la recomposition algorithmique des textes. C'est le cas d'un des livres du catalogue, $L^{\prime}$ art des dilutions ${ }^{33}$, transvasé sur une page html qui recourt à un script JS et au glitch-art ${ }^{34}$ pour créer un dispositif interactif permettant différentes trajectoires de lecture d'un recueil d'agit-prop "griffonné sur la N113, entre deux ronds-points" qui se reconnait "d'une poétique des Gilets Jaunes"35. Les utilisateur.trice's remarqueront aussi que l'ensemble des "antilivres" de la maison d'édition, qu'il s'agisse des versions mises en forme pour la publication papier ou des dispositifs numériques, sont publiés accompagnés d'un répertoire github qui permet à chacun'e d'avoir accès au code, de le modifier et de le réutiliser. On sait combien la dématérialisation aide à rendre la propriété privée ingérable et ces décisions sont cohérentes avec la mise à disposition des textes sous des licences libres Creative Commons: deux choix éditoriaux qui inscrivent la démarche dans les philosophies et les pratiques open-source, caractéristiques des idéologies cyberanarchistes déjà mentionnées.

8 Ainsi, en dehors de toute politisation immédiate du code, certains collectifs commencent à exploiter les possibilités des littératures numériques à des fins militantes. On peut déjà en identifier deux : la recherche d'une nouvelle performativité des dispositifs et l'inscription dans les configurations attentionnelles apparues avec les nouveaux médias. Toutefois, il s'agit encore de rénovations formelles des écritures militantes qui ne s'attèlent pas nécessairement à une politisation de l'ensemble des textualités natives du web, devenues aujourd'hui de nouvelles zones à défendre (et à attaquer). 


\section{Architextes du contrôle}

9 Les théoricien-ne's de la cybersphère insistent beaucoup sur son hypertextualité, soit le fait qu'elle est un ensemble de textes prévoyant une pluralité d'itinéraires possibles, un système qu'on pourrait efficacement décrire comme une bibliothèque "multiséquencielle" ${ }^{36}$ (un usage du term distinct donc, de celui auxquels les littéraires sont habitué.e.s). Mais la notion d'hypertexte ne prend en compte que ce que les développeur-euse's appellent le front end, les textes visibles mis en réseau et accessibles les uns aux autres selon un rapport (qu'on décrit parfois à tort comme a-hiérarchique). C'est oublier que l'hypertexte entretient un rapport de dépendance au code qui le régule, le back end, qui est également un assemblage de langages. En considérant que le code est un texte, l'hypertexte n'est que l'aspect visible d'un architexte:si le web apparait à nombre d'utilisateur-trice's comme un réseau d'informations en langage naturel (le français, l'anglais, etc.), la totalité de ce langage est sous-déterminée par un réseau de langages qu'on appelle formels ${ }^{37}$. Il suffit pour le comprendre d'ouvrir n'importe quelle page web, d'effectuer un clic droit et de sélectionner la commande Inspecter ou Afficher le code source de la page ${ }^{38}$. Le document qui s'ouvre alors est un fichier rédigé dans un ensemble de langages formels compilés et interdéterminés les uns par les autres. Ils peuvent décrire des règles d'affichage, gérer l'interaction de l'utilisateur avec la page ou soutenir des scripts algorithmiques.

Le web est donc un architexte au sens d'un assemblage complexe de langages naturels et d'images régulés par une couche de langages formels empilés qui le constitue comme dispositif et comme espace-temps (on pourrait presque dire, comme expérience). Ce point est essentiel dans la mesure où l'hypertexte, et l'ensemble du web comme dispositif, est inféodé à l'architexte. Dans une perspective computationnelle, l'architexte fait du net un système de guidage par le langage, du point de vue de Lessing:

La vie dans le cyberespace est régulée avant tout à travers le code du cyberespace. Régulée dans le sens où les barres d'une prison régulent le mouvement d'un prisonnier, ou régulée dans le sens où des marches régulent l'accès d'un espace aux personnes atteintes de handicap. Le code est le régulateur du cyberespace parce qu'il définit les termes selon lesquels le cyberespace est offert. Et celles et ceux qui fixent ces termes reconnaissent de plus en plus le code comme le moyen de guider les comportements qui leur bénéficient le plus. ${ }^{39}$

11 Ce constat souligne combien la dissimulation croissante des codes sources constitue une invisibilisation des infrastructures du contrôle : plus on coupe les utilisateur.trice's du code, plus on tend à rendre le système de guidage imperceptible. Il n'est d'ailleurs pas surprenant que les factions impliquées dans les cyberluttes envisagent la connexion architexte-utilisateur.trice dans des perspectives très différentes. Les régulateur.trice-s du trafic - en position dominante (les GAFAM, mais aussi les états et leurs cyberpolices) - prônent un web user-friendly: le dogme marketing dominant exige des interfaces flexibles et simples d'utilisation, toujours plus éloignées des fonctionnements encodés de l'architexte, soit, dans la plupart des cas, du fonctionnement réel des protocoles de guidage. La hacksphère, de son côté, prône une longue et douloureuse auto-éducation à des systèmes libres comme Linux qui engagent chaque utilisateur.trice à comprendre patiemment les rouages des programmes avant de les utiliser, de même qu'une esthétique minimale rêvant parfois une transparence entre front end et back end dont atteste par exemple l'usage de l'ASCII ART ${ }^{40}$. 
12 Dans une perspective post-structuraliste comme celle de McKenzie Wark, théoricienne essentielle du hack et notamment autrice du Hacker Manifesto ${ }^{41}$, il n'y a pas de différence entre le contrôle et le dispositif de contrôle. Wark accorde une importance particulière au langage, qu'elle considère doublement comme manifestation rhétorique de l'autorité (représentation du pouvoir via le langage naturel), mais aussi comme dispositif encodant au cœur de l'autorité computationnelle : le langage autoritaire, comme le texte de loi, constitue une nouvelle strate de code à même de guider les comportements et les croyances. Partant, la complexification croissante du langage juridique, comme celle de l'architexte, participeraient d'un même mouvement déconnectant les dispositifs de contrôle de celles et ceux à qui on les impose.

13 La structure architextuelle n'est pas une réalité autoritaire en soi, il semble plus juste de dire qu'elle est le lieu d'une lutte et que les instances dominantes ne cessent de réinventer de nouvelles zones et de nouvelles configurations autoritaires de l'architexte, observables à différentes échelles. Par exemple, pendant l'écriture de cet article, alors que les cellules de défense du Rojava (Kurdistan autonome) s'organisent sur le terrain et en ligne, nombre d'hacktivistes occidentaux traquent et détruisent d'autres cellules, les turkbots (à distinguer des "AK Trolls") sur les réseaux sociaux. Ces bots (similaires à ceux de la maison Abrüpt mais au service d'intérêts contrerévolutionnaires) sont des programmes automatiques codés pour traquer les publications pro-Kurdes des utilisateurtrice's, les signaler ${ }^{42}$ et les couvrir de commentaires favorables à la politique répressive d'Erdogan ${ }^{43}$. Autre exemple plus systémique: la suggestion algorithmique sur laquelle reposent toujours plus des mastodontes comme Facebook et Youtube. En contexte médiarchique, la rhétorique autoritaire d'un discours de Donald Trump est compréhensible avec les outils développés pour l'étude des langages naturels (comme texte), lorsqu'elle s'intègre dans l'architecture des pages de Facebook (comme hypertexte) organisée algorithmiquement par le code du site (comme architexte), on peut donc difficilement ignorer le cadre attentionnel du message et les codes qui le régulent. Le fait qu'un même discours soit re-suggéré et pluralisé au moyen d'un algorithme préférentiel change la donne ${ }^{44}$. La cybersphère produit donc l'expérience visuelle et cognitive de multiples protocoles de guidage imbriqués, effectifs à différents niveaux de visibilité et d'interactivité, qu'on peut alors déjouer ou pirater sur des plans distincts.

Cet interlude théorique pour en arriver au constat suivant: que le fondement du cybercontrôle soit un ensemble de textes en langage formel autorise à rêver une écriture-action-directe qui, réécrivant l'architexte, réécrit immédiatement le système de contrôle - mais il faut garder à l'esprit que cette cyberlittérature est encore essentiellement un rêve. Pour autant, plusieurs auteur'trice's et hackeur'euse's investissent cette interdétermination comme lieu d'écriture où l'on manipule, non seulement les langages naturels (comme les écrivaine's) ou les langages formels (comme les hackeur·euse·s), mais les deux en même temps. Plus exactement, puisque les possibilités de l'architexte sont majoritairement utilisées aujourd'hui pour régénérer le contrôle, il faudrait dire qu'il·elle's entendent proposer des contre-dispositifs, des écritures contestataires de l'architexte qui s'attaquent aux écritures dominantes. Suivant les deux fonctions traditionnellement attribuées aux contre-récits militants, révéler l'oppression et réinventer la désobéissance, on voudra présenter ici deux démarches complémentaires: les projets qui entendent dénoncer les architextes autoritaires et ceux qui entendent les hacker. 


\section{Dénoncer les architextes autoritaires}

15

Explorer une écriture à l'interaction des langages, par militantisme ou simplement par jeu, a toujours été indissociable des contre-cultures numériques. L'histoire du hack a gardé trace d'un projet décrit dans un fanzine de passionée's d'Amiga et resté célèbre sous le nom de Rendering Speeches. Dès 1993, le polonais Marek Pampuch commence à développer un programme qui génère de manière algorithmique des discours de propagande communiste, à partir de séquences sélectionnées dans l'interdiscours historique ${ }^{45}$. Speeches - originellement une boutade satirique - constitue un exemple déjà ancien d'écriture politique inscrite à l'interaction des langages formels et naturels. Le principal intérêt de Speeches est de permettre à l'utilisateur.trice de générer une infinité de discours politiques et donc de produire une expérience de lecture dans laquelle la rhétorique du pouvoir se virtualise infiniment. Le premier discours généré parait sensé, mais à chaque réitération de l'opération demandée par l'utilisateur.trice, les configurations de la parole autoritaire paraîtront plus absurdes, les structures devenant répétitives et identifiables. Si le programme de Pampuch n'a pas l'ambition d'une praxis réfléchie, il souligne que la temporalité et l'interactivité du dispositif peuvent faire du code un lieu d'encapacitation. La petite cellule architextuelle qu'est Speeches entend rendre à chacun'e une maîtrise, voire un contrôle, de la rhétorique totalitaire : l'expérience interactive de ses répétitions et de ses vacuités souligne que l'on peut faire de la génération algorithmique un lieu de renversement des positions de pouvoir.

Dans une perspective inverse, depuis 2014, le programmeur et artiste mexicain Eugenio Tisselli se livre à un démontage plus complexe dans le projet de longue haleine The 27th / El 27, caractéristique de la transition opérée sur le net entre art comme œuvre et art comme processus ${ }^{46}$. Sur une page web ${ }^{47}$, Tisselli a réécrit en espagnol l'article 27 de la constitution mexicaine. Censé protéger les territoires indigènes et les modes d'existence communaux, l'article stipule que toutes les terres du pays sont la propriété du peuple, mais il a été considérablement amendé après la signature du NAFTA (North American Free Trade Agreement) pour permettre la mise en place d'infrastructures destinées à l'accueil de touristes (principalement états-unien-ne-s). Depuis, sur le site de Tisselli, chaque jour où l'index de Wall Street se clôt à la hausse, un mot de l'article 27 est traduit en anglais, précisément dans un anglais distordu par des algorithmes. En 2019, il ne reste bientôt plus un seul mot en espagnol sur la page du projet $E l ~ 27$ (ce qui en dit long sur la bonne santé de la finance internationale). Comme chez Pampuch, on retrouve chez Tisselli la volonté d'une écriture directement inscrite dans la complexité des architextes autoritaires. Dans les termes de l'artiste :

La dictature financière mondiale nous présente un paradoxe: si les transactions économiques capables de changer le destin de pays entiers résultent d'un langage performatif, c'est le langage lui-même qui, à son tour, est transformé et soumis aux flux des marchés financiers. ${ }^{48}$

Outre la mise en texte et en durée du colonialisme américain dont sont victimes les populations mexicaines, le travail sur les langages naturels d'El 27 explicite ce que les rapports de pouvoir internationaux peuvent avoir de textuel (ou de textualisable), en soulignant l'inégalité entre les traités de libre-échange internationaux et les lois populaires. La dénonciation du capitalisme computationnel (Tisselli revendique le terme), passe alors par la mise en évidence de la mutation algorithmique du pouvoir: 
"L'érosion du sol et de la culture mexicains par le commerce algorithmique ultrarapide et les robots linguistiques finira par se terminer dans un paysage textuel non humain de non-sens spéculatifs" ${ }^{49}$. Mais le dispositif d'El 27 veut aussi inscrire les langages formels dans le rapport de force, d'une manière opposée à ce qu'on a pu observer dans Rendering Speeches. Les deux projets travaillent sur l'architextualité, mais la relation de pouvoir entre les langages est inversée ${ }^{50}$. Dans le cas des Speeches, la page web place le langage formel en position dominante, puisque le dispositif procédural rend aux lecteur.trice's un pouvoir sur la rhétorique politicienne, qu'ils et elles peuvent regarder dépérir jusqu'à l'absurde au fur et à mesure que l'algorithme est relancé. Chez Tisselli, au contraire, les lecteurtrice's sont impuissante's face à la disparition programmée de la version espagnole, impuissance qui entend répliquer celle du peuple mexicain face à la colonisation. Ne réagissant qu'aux transactions algorithmiques de Wall Street, le langage formel - le code source de la page - est déconnecté des lecteur.trice's. Et les lecteur-trice's sont déconnectée's du langage formel, d'où une transformation du dispositif encapacitant de Pampuch en un dispositif aliénant. Là où l'un entend renverser le rapport de contrôle, l'autre le révèle et ce faisant, le dénonce. Sans surprise, les presque vingt années qui séparent les deux projets témoignent en creux de la reconfiguration des rapports de pouvoir, mais aussi d'un imaginaire distinct sur le pouvoir des algorithmes. Si l'on lit dans Speeches l'ironie d'un tech-enthusiast sur une rhétorique politicienne perçue comme obsolète, El 27 témoigne d'une prise de conscience de l'autorité algorithmique au XXIe siècle et d'un recul critique sur les utopies digitales ${ }^{51}$.

Notons que ces exemples se contentent d'exposer les architextes, sans prétendre constituer une action. Le dispositif architextuel sert une dénonciation, elle est utilisée comme on utilise le papier imprimé pour diffuser sa propagande ou comme on utilise une galerie pour y proposer une installation militante. Ainsi, Pampuch et Tisselli attestent bien de la possibilité de dispositifs hacktivistes littérarisants qui révèlent et dénoncent les architextes autoritaires, mais ils n'entendent pas proposer une praxis à proprement parler.

\section{Hacker les architextes autoritaires}

19 Les principales acceptions anarchistes de la praxis font de l'action directe le pendant de l'activité non moins nécessaire de propagande (manifestations, tractage, affichage, etc.). Avec l'extension du domaine du hack théorisée par Wark et d'autres, les praxis hacktivistes ne manquent pas. Comme le soulignent Palma et Von Bush ${ }^{52}$, elles peuvent être pensées dans une perspective destructrice (attaquer les protocoles qui condensent l'information et le pouvoir) ou constructrice (inventer de nouveaux modes d'organisation). On repense ici aux déjà évoquée's urban hackers qui transforment les bancs publics en refuges pour les personnes sans-abri ou aux gender-hackers qui "piratent" leurs propres corps pour contourner les discriminations administratives et médicales subies par les personnes trans.

En théorie comme en pratique, certaines écritures essaient de sortir de la seule logique de la dénonciation pour constituer immédiatement des actes. Ces pratiques sont aussi protéiformes que le web lui-même, mais on peut interroger certaines de leurs conditions de possibilité. On propose d'en identifier trois : $1 /$ une écriture hacktiviste doit proposer une littérarité qui agit directement sur les architextes autoritaires ; 2/ en 
conscience de l'interactivité des dispositifs ; 3/ en s'insinuant directement dans les configurations attentionnelles fabriquées par nos médiarchies "qu'il est leurrant d'imaginer comme des démocraties" 53 . Si l'on voulait faire écho à d'autres classifications esthétiques, il conviendrait sans doute de dire que les écritures hacktivistes sont un sous-genre spécifiquement textuel (ou littéraire) de ce qu'Yves Citton appelle justement les médiartivismes, des projets qui prennent les structures et les relations médiatiques comme matériau artistique principal.

21 L'une des possibilités de ces hacktivismes littéraires est naturellement d'inscrire leurs écritures dans l'hyperactivité des communications contemporaines. C'est en tout cas la piste qu'a explorée le projet ScareMail ${ }^{54}$ conçu par le prolifique Ben Grosser comme une action directe contre la surveillance globale. Actif pendant plusieurs années (mais malheureusement vulnérable aux mises à jour), ScareMail est un plug-in fonctionnant sur les principaux navigateurs qui ajoute en signature de chaque mail envoyé par l'utilisateur.trice un extrait du roman Farenheit 451 truffé de mots clés susceptibles d'attirer l'attention des programmes de pistage de la NSA. On sait aujourd'hui que, sous prétexte de lutte antiterroriste, près d'un tiers des communications numériques mondiales sont stockées et écoutées par les services de renseignements américains qui, pour filtrer une quantité ingérable de données, utilisent des programmes signalant les communications à risque en identifiant des mots-clés préalablement listés. Suite au hack de certains services de renseignement ${ }^{55}$, plusieurs de ces listes ont été révélées, permettant à ScareMail de voir le jour. Lorsque le dispositif de Ben Grosser insère automatiquement nombre de ces mots-clés (de manière visible ou invisible) dans les signatures, chaque mail envoyé par l'utilisateur.trice fait perdre du temps aux programmes de la NSA en les forçant à traiter une requête inquiétante, mais factice. ScareMail a toutefois dû trouver un moyen de déjouer le filtrage automatique des algorithmes de surveillance, capables de détecter et d'ignorer les structures répétitives et autres formes de données textuelles qu'on peut identifier comme d'origine nonhumaine ${ }^{56}$. C'est ici que la littérature entre en jeu. Ben Grosser a conçu son propre algorithme grâce aux protocoles de reconnaissance des langages naturels qui identifient dans Farenheit 451 les noms et les verbes pour les remplacer par les mots-clés inquiétants. La virtualisation et la pluralisation d'un texte d'origine unique permet à chaque mail d'inclure (automatiquement) un extrait transformé constituant un récit plausible, grammaticalement correct, impossible à attribuer à une machine et suffisamment unique pour que les bots de la NSA recommencent leur travail d'inspection à chaque envoi de message contenant ces pastiches de littérature sciencefictionnelle.

L'artiste a estimé que si 10\% des internautes utilisaient l'extension, les algorithmes de surveillance, aux résultats déjà fragiles, deviendraient complètement inutiles. ScareMail constitue un exemple d'écriture dont la textualité même relève de l'action directe, unique sous de nombreux aspects. Le plus étonnant est peut-être que le dispositif prévoit d'abord et avant tout des lectures non-humaines et, pour déjouer ces inspections autoritaires, n'a aucunement besoin que les histoires en langage naturel soient visibles. L'automatisation des processus permet alors à une écriture hacktiviste d'être immédiatement performative - encore une fois, performative dans un espace surveillé dont le langage formel est la réalité - et à sa puissance d'action d'être démultipliée par la quantité d'utilisateur.trice's et la quantité d'e-mails que nous envoyons quotidiennement. 
23 S'il est complexe d'inventer des écritures dont la textualité soit une action directe, ScareMail n'est pas un cas isolé. Utiliser l'hyperconnectivité contemporaine comme puissance multipliant l'énergie d'une action est au cœur d'autres projets hacktivistes, comme les travaux de Bret Stalbaum autour des virtual sittings. Le terme vulgarise ce que la hacksphère connait sous le terme de Distributed Denial-of-Service attack (DDoS) : une attaque dont l'objectif est de saturer le serveur ciblé de requêtes jusqu'à le rendre inutilisable ${ }^{57}$. Si l'endurance des serveurs dans les années 2000 permettait de mener ces attaques humainement, en demandant par exemple à des centaines d'activistes de se connecter de manière répétée à un site victime, elles ne sont plus aujourd'hui efficaces qu'avec des programmes qui actualisent infiniment une page ou avec des multitudes d'agents zombies: les hacktivistes piratent les connecteurs de n'importe quel appareil relié au web et reroutent automatiquement des milliers (voire des dizaines de milliers) de requêtes sur le site ciblé, le désactivant le plus longtemps possible. Pour démocratiser les DDoS et les transformer en dispositifs d'écritures militantes collectives, Stalbaum et un collectif d'artistes et de programmeur.euse's mettent en place Flood Net ${ }^{58}$ dans les années 90 et l'améliorent jusqu'à son abandon en 2008 faute de relève. FloodNet est un générateur dans lequel chacun'e peut sélectionner une cible, le site web d'un service gouvernemental ou d'une entreprise par exemple, et déclencher immédiatement une attaque DDoS. Plusieurs fonctionnalités du projet permettent à Stalbaum de parler "d'art conceptuel" et "d'écriture collective" ${ }^{59}$. La principale singularité de FloodNet est de permettre à celles et ceux qui commanditent une attaque de rentrer un message personnel qui, dans le meilleur des cas, s'affichera en lieu et place de la page désactivée. Dans le cas de sites à l'infrastructure assez faible, certain·es activistes ont pu remplacer pendant plusieurs heures les pages d'accueil par des messages de propagande hostiles aux corporations visées, ou des poèmes de leur cru. Stalbaum recense également certains usages des ressources d'affichage html, comme la possibilité de programmer une requête pour human_rights, entraînant le remplacement de certaines pages par le message automatisé human_rights not found on this server. Transvasés dans un contexte réel, FloodNet équivaudrait à un protocole permettant de recouvrir provisoirement un bâtiment symbolique d'un immense drap blanc sur lequel chacune aurait écrit le message de son choix. Comme dans le cas de ScareMail, l'écriture en langages naturels participe à l'efficience de l'action parce qu'elle produit un texte spécifique qui déjoue les protocoles de reconnaissance automatique des requêtes mis en place pour défendre les serveurs : chaque message personnalisé engage à réitérer la vérification de zéro, alors qu'une multitude de connexions automatisées peuvent être ignorées par les pare-feu contemporains. Stalbaum et ses complices rapprochent FloodNet des écritures urbaines illégales, prônant l'usage de textes littéraires comme EGraffiti. En atteste une action lors de laquelle il·elle's ont utilisé le programme pour envoyer une masse de requête ingérables sur le serveur du président mexicain d'alors, remplaçant les messages d'erreur html par des extraits de Don Quichotte, réécrits et croisés avec des lignes de certains communiqués révolutionnaires zapatistes ${ }^{60}$. Un protocole d'action comme FloodNet enregistre lui aussi la nécessité d'une réinvention des luttes à l'ère de la domination "liquide" 61 , proposant non une action individuelle isolée, mais un dispositif de désobéissance permettant à tous·te·s de venir réécrire les interfaces numériques $\mathrm{du}$ pouvoir. Ce cybersitting permet d'envisager une désobéissance à l'intérieur de l'architexte, un hack direct, non d'un texte ou d'un espace, mais du nexus entre les langages et les attentions qui constitue les interfaces. En cela, FloodNet intègre une dimension artistique aux doctrines de ce qu'on appelle la 
désobéissance civile électronique dont l'idée principale est que la décentralisation et la dématérialisation numérique du pouvoir rendent de plus en plus ineffectives les actions physiques, comme les blocages de rue :

La désobéissance civile traditionnelle n'a plus d'effet sur le cœur de l'organisation, au contraire, elle tend à se concentrer dans une structure sédentaire localisée. [...] Dominer physiquement les sites stratégiques était autrefois la source du pouvoir, mais maintenant la domination s'appuie sur l'aptitude des institutions à se déplacer là où la résistance est absente, en conjonction avec sa capacité à se réapproprier en permanence les espaces physiques dont elle a besoin. ${ }^{62}$

Si l'extension du domaine de la littérature au numérique est aujourd'hui un champ d'étude et d'expérimentation majeur pour les auteur.trice's et les chercheur.euse-s, les usages politiquement radicaux qu'on peut en faire sont inégalement connus et explorés. Sans prétendre à aucune exhaustivité, il a semblé pertinent de souligner ici deux types de pratiques distincts dans leurs enjeux médiaux et politiques. D'un côté, on observe que certains collectifs entendent rénover les codes et les objectifs des littératures radicales traditionnelles, qu'il s'agisse de leurs formes ou de leurs connexions médiales. De l'autre, certain'e's écrivain'e-s-hackers fondent leur geste d'écriture sur la nature architextuelle du web, consciente-s que les rapports de pouvoir sur la cybersphère sont largement inscrits dans les rapports de déterminations entre les langages naturels et les langages formels. Ces pratiques d'écriture dans les deux langues peuvent dénoncer les architextes autoritaires ou essayer de constituer une action directe visant à les hacker. En révélant que le cybercontrôle repose avant tout sur des processus textuels, ces écritures hacktivistes se donnent pour horizon d'inventer des réécritures $d u$ contrôle. Parmi les conditions de possibilités d'une littérature hacktiviste identifiées ici, les plus déterminantes semblent: la constitution de nouveaux architextes (l'écriture dans les deux types de langage), la mise en place de contre-dispositifs (interactifs et cherchant une nouvelle performativité) et l'inscription dans les configurations attentionnelles induites par les nouveaux médias. Les formes classiques de contrôle ont la réputation d'avoir toujours un temps de retard sur l'inventivité militante, mais la cybersphère et l'entrée dans le jeu du pouvoir technopolitique des GAFAM change la donne : le cybercontrôle se complexifie de plus en plus vite, et les sociétés contemporaines déconnectent toujours plus les netizens du code qui régule le pan numérique de leur existence. Un constat qui ne peut sonner que comme une invitation à réinventer sans cesse les luttes du cyberespace - une réinvention technique, mais aussi artistique, où la littérature, à n'en pas douter, a son rôle à jouer.

\section{NOTES DE FIN}

1. Acronyme couramment utilisé pour désigner les puissances économiques, mais aussi politiques, de la Silicon Valley : Google Amazon Facebook Apple Microsoft.

2. G8 de l'internet, organisé à Paris à l'initiative de Nicolas Sarkozy comme préambule au $37^{\mathrm{e}}$ sommet du G8.

3. Qu'on entend ici en filiation avec Foucault, soit dans le sens d'une normalisation des comportements et des pensées par intériorisation individuelle et mentale de la surveillance. On 
considère souvent que le contrôle est largement régénéré par les nouvelles technologies et notamment par les protocoles de guidage des attentions qu'on évoquera ci-après.

4. Jean Cristofol, "Écritures, dispositifs et expériences", dans Colette Tron, Emmanuel Vergès (dirs), Nouveaux médias, nouveaux langages, nouvelles écritures, Vic La Gardiole, éditions l'entretemps, 2005, p. 24.

5. Sur les distinctions entre capitalisme cognitif et capitalisme computationnel, voir notamment David Berry, "Subjectivités computationnelles", Multitudes, n59, Humanités numériques 2.0, 2015.

6. En français, on trouve une synthèse critique sur le sujet dans Yves Citton, Pour une écologie de l'attention, Paris, Seuil, 2014.

7. Sur les nouvelles technologies comme vectrices d'auto-organisation et la fonction expressiviste des médiactivismes, voir Dominique Cardon, Fabien Granjon, Médiactivistes, Paris, Presses de Sciences Po, p. 13-23 et 81-110.

8. Un terme de plus en plus utilisé, notamment au Québec, pour problématiser la manière dont le cyberespace interagit avec le monde réel, voir la définition qu'en propose Jean-François Legault, Manipulation de code et avant-garde: pour une littérature hackée, Montréal, Université du Québec à Montréal, 2012, p. 4.

9. Collectif Mauvaise Troupe, Constellations. Trajectoires révolutionnaires du jeune XXIe siècle, "Et si le monde du logiciel libre prenait parti ?", accessible en ligne, url : <https://mauvaisetroupe.org/ spip.php?article84>.

10. Sur le sujet voir le volume essentiel de Brian Alleyne, Geek and Hacker Stories. Code, Culture and Storytelling from the Technosphere, Londres, Palgrave MacMillan, 2018; ainsi que le plus ancien Katie Hafner, Matthew Lyon, Where Wizards Stay Up Late. The Origins of the Internet, New York, Touchstone, 1996.

11. Une notion précisément explicitée par Brian Massumi, L'économie contre elle-même : vers un art anti-capitaliste de l'événement, Paris, Lux, 2018.

12. Richard Stallman, un des pionniers du cyberanarchisme raconte par exemple la guerre perdue par ces premier.ère.s hackeur.euse.s contre le principe même du mot de passe dans Sylvain Bergère, Une contre histoire de l'internet, Arte, 2013.

13. Dont Stallman est un initiateur, pour une étude historique approfondie, voir Glyn Moody, Rebel Code : Linux and the Open Source Revolution, Londres, Basic Books, 2002.

14. Détournement des technologies numériques pour désorienter les dispositifs de surveillance policière durant les manifestations (riot), ainsi, durant la rédaction de cet article, les manifestant.e.s hong-kongais.es utilisent des t-shirts imprimés de visages célèbres pour invalider la reconnaissance faciale ou des lasers de poche pour aveugler les drones de la police ; on trouve une liste d'idée DIY dans Geoffrey Dorne, Hacker Citizen, Paris, Tind!, 2016.

15. Désigne globalement le détournement de dispositifs technologiques pour contourner les discriminations de genre, voir notamment le Pechblenda Lab en Espagne, et les initiatives qu'ils multiplient autour du féminisme transhack.

16. Le détournement contestataire des infrastructures urbaines, ainsi l'artiste français Florian Rivière transforme les bancs publics anti-sdf en abris à l'aide d'une perceuse ; c'est aussi un axe de réflexion du SenseLab américain, voir Brian Massumi, Erin Manning, Pensée en acte. Vingt propositions pour la recherche création, publié à l'initiative d'Yves Citton, Paris, Les presses du réel, 2018, dont j'ai eu l'occasion de discuter les enjeux politiques dans Aurélien Maignant, "Auto(dés)organisation : recherche, création \& activisme au SenseLab”, Acta Fabula, vol. 20, n8, 2019.

17. Andrew Murphie, "Hacking the Aesthetic : David Haines and Joyce Hintering's New Ecologies of Signal", Journal of Aesthetics \& Culture, vol. 4, n¹, 2012.

18. Pratique consistant à modifier des sons et des images fixes ou animées en ingérant directement dans le code des fichiers. 
19. Sujet d'innombrables travaux, une étude sociologique en français sur les enjeux politiques des arts numériques a été proposée par Paul Fourmentraux, L'œuvre virale. Net art et culture hacker, Bruxelles, La Lettre volée, 2013.

20. Une comparaison qui permettrait de comprendre le hack par la peinture et la peinture par le hack selon Paul Graham, Hackers \& Painters. Big Ideas from the Computer Age, Sebastopol, O'Reilly Media, 2004.

21. Par Jean-François Legault, op. cit..

22. On revient plus tard sur l'importance du terme de dispositif, voir la défense du terme pour préciser les conditions d'existences atypiques des écritures contemporaines, voir Christophe Hanna, "La métaphore ufologique", Nos dispositifs poétiques, Paris, Questions théoriques, 2010, 1-24.

23. On pense notamment à la perspective proposée par un hacktiviste historique, Oxblood Ruffin, "Hacktivism and Speech Rights, Then and Now", Modalities of Revolt, Columbia, Columbia University, 2018. Pour une approche universitaire récente des activismes numériques citoyens, voir Clegg S. Ghobadi, "these days will never be forgotten : Critical Mass Approach to Online Activism", Information and Organization, vol. 25, n¹, 2014, p. 2-71.

24. Dominique Cardon, Fabrice Granjon, op. cit..

25. Comme l'impressionnant The Anarchist Library. Url : <https://theanarchistlibrary.org/special/ index>.

26. Laboria Cuboniks, Xenofeminist Manifesto. Url : <http://www.laboriacuboniks.net/>.

27. Elle est traitée notamment chez Jean-François Legault, op. cit., p. 96-98 et Otto Von Busch, Karl Palmås, Abstract Hacktivism. The Making of a Hacker Culture, OpenMute, 2006, p. 39-45.

28. Notamment par Serge Bouchardon, La valeur heuristique de la littérature numérique, Paris, Hermann, 2014, p. 52-61.

29. Serge Bouchardon, Siyu Zhang, Xuhao Lin, Romaric Delahaie, Rebecca Fribourg, Raphaël Kovacic, Agir, application mobile, 2016, url : <http://sergebouchardon.com/>.

30. D'après le descriptif de la maison d'édition. Url : <http://www.abrupt.ch/>.

31. On utilise le terme dans le sens que lui donne Yves Citton, Médiarchies, Paris, Seuil, 2017. Il s'agit de visibiliser le rôle que jouent les formes des médias (caractérisées par une recherche permanente d'invisibilité) dans l'exercice du pouvoir. Le rapport de pouvoir médiarchique se donne essentiellement comme oligarchique, même si leur redéfinition technique et politique permanente et suivant des intérêts en conflit, incite Citton à privilégier l'idée d'une hétérarchie (pp. 50-55).

32. Régulièrement pris pour cibles par les médiartivistes, voir par exemple les différents demetricator et le SafeBook de Ben Grosser.

33. Pier Lampás, L'art des dilutions, Zürich, antilivre dynamique, Abrüpt, 2020, url : <https:// txt.abrupt.ch/antilivre/abrupt_lampas_pier_lart_des_dilutions_antilivre_dynamique.html>.

34. Ensemble de pratiques explorant les possibilités esthétiques des disfonctionnements et des bugs analogiques ou numériques, parfois employées dans l'intention militante de révéler les dispositifs de contrôle invisibilisés : "nos appareils de médiation tendent à rester invisibles ou transparents tant qu'ils fonctionnent selon ce que nous attendons d'eux. Ils ne s'imposent pleinement à notre attention [...] que lorsqu'une panne fait apparaître la dépendance que nous avons développée à leur égard" d'après Yves Citton, Médiarchies, Paris, Seuil, 2017, p. 350.

35. D'après “L'art des dilutions - Pier Lampás", lundimatin, \#225, janvier 2020, url : <https:// lundi.am/L-art-des-dilutions-Pier-Lampas>. Pour une étude de la poétique à l'œuvre dans les slogans des gilets jaunes, voir Denis Saint-Amand, "Parce que c'est notre rejet. Poétique des gilets jaunes", AOC, 2 février 2019, url : <https://aoc.media/analyse/2019/02/01/cest-rejet-poetiquegilets-jaunes/>.

36. Voir notamment la discussion technique autour des implications pour la lecture qu'en donne Alain Giffard, "Idée du lecteur", dans Colette Tron, Emmanuel Vergès (dirs), op. cit., p. 84-85. 
37. L'essentiel des projets connus utilisent principalement la triade html, css, javascript (et dérivés), mais il existe une grande quantité de langages encodants. Désigner l'ensemble de ces langages non-naturels comme "formels" est une simplification volontaire. Si les ordinateurs et les pages web fonctionnent par couches de code (du langage machine à l'utilisateur via plusieurs étapes de traduction et de compilation), on peut se satisfaire ici d'un modèle en deux temps, notamment sur le net. On se contentera de l'expression "langage formel" (ensemble des systèmes de codage), comme pendant aux "langages naturels" (vecteurs de l'information compréhensible par tous.tes comme l'anglais, le français, etc.), car on verra que la dichotomie suffit déjà à saisir nombre de tensions sociales, politiques et attentionnelles.

38. Sur Mac avec le raccourci clavier $\mathrm{Cmd}+\mathrm{U}$ ou $\mathrm{Ctrl}+\mathrm{U}$ sur PC.

39. Je traduis. Laurence Lessig, Code, New York, Basic Books, 2006, p. 83-84 : "Life in cyberspace is regulated primarily through the code of cyberspace. [...] Regulated in the sense that bars on a prison regulate the movement of a prisoner, or regulated in the sense that stairs regulate the access of the disabled. Code is a regulator in cyberspace because it defines the terms upon which cyberspace is offered. And those who set those terms increasingly recognize the code as a means to achieving the behaviors that benefit them best."

40. Type d'art figuratif basé sur les caractères de la norme ASCII plébiscité par la hacksphère pour son minimalisme, mais surtout parce qu'elle permet de réaliser des images immédiatement en langage formel et naturel, voir par exemple la page d'accueil du site d'une communauté de hackeur.euse.s comme Cult of the Dead Cow et lancer la commande "afficher le code source". Url : $<$ https://cultdeadcow.com/>.

41. McKenzie Wark, A Hacker Manifesto, Cambridge, Harvard University Press, 2004.

42. Soit les dénoncer aux hébergeurs comme inappropriées, ce qui implique un protocole de traitement pouvant mener à leur suppression, et à la suspension provisoire ou définitive des comptes.

43. Dont le parti est l'APK, d'où l'acronyme "AK Trolls" parfois utilisé pour parler des bots, alors que deux réalités distinctes sont en jeu: des programmes qui ont un comportement politique automatisé et des personnes réelles rémunérées pour diffuser la propagande du gouvernement turc sur le net. Voir notamment la recension qu'en donne Medium, url : <https://medium.com/ dfrlab/bot-like-turkish-accounts-wage-anti-kurdish-hashtag-campaign-9b1a2908f5b3>

44. On sait aujourd'hui combien ces logiques de re-suggestion algorithmique sont déterminantes pour la construction des croyances, notamment en période électorale.

45. La version contemporaine est curatée en ligne, Marek Pampuch, Renderign Speeches, Cura : A Literary Magazone of Art \& Action, n¹4, 2014 [1993-1997], url : <http://curamag.com/issues/ 2014/12/3/renderings-speeches> et ELO : <http:/collection.eliterature.org/3/works/speeches/ en.html>.

46. Une des thèses développées par Paul Fourmentraux, op. cit.; Eugenio Tisseli, The 27th / El 27, galerie virtuelle sur le site personnel de l'artiste, 2014, url : <http://motorhueso.net/27/>.

47. http://motorhueso.net/27/

48. Je traduis: "[t]he global financial dictatorship presents us with a paradox: while the economic transactions capable of shifting the destinies of entire countries are the result of performative language, it is language itself that, in turn, is transformed and subjected to the flows of financial markets"; Eugenio Tisselli, El 27 / The 27th, sur collection.eliterature.org, 2013, url : <http://collection.eliterature.org/3/work.html?work=the-27th>.

49. Je traduis: "The erosion of Mexican soil and culture at the hands of ultrafast algorithmic trading and linguistic robots will eventually terminate in a nonhuman textual landscape of speculative nonsense"; Eugenio Tisselli, op. cit., url: <http://collection.eliterature.org/3/ work.html?work=the-27th>.

50. "L'une des fonctions essentielles d'un dispositif est bien de situer la place de chacun dans un [...] rapport de force. Ce que les dispositifs mettent en jeu, ce sont les modalités du voir et du 
discourir, les conditions de l'exercice du pouvoir et la constitution de soi."; Jean Cristofol, "Écritures, dispositifs et expéricences", dans Colette Tron, Emmanuel Vergès (dirs), op. cit., p. 17. 51. Voir également les autres projets de la section "algorithmic politics" de motorhueso, le site de Tisselli. Url : <http://www.motorhueso.net/>.

52. Otto von Busch, Karl Palmå, op. cit..

53. Yves Citton, Médiarchies, Paris, Seuil, 2017, p. 13.

54. Ben Grosser, ScareMail, extension de navigateur, hébergé sur une galerie personnelle, 2013, url : <https://bengrosser.com/projects/scaremail/>.

55. L'existence de ces listes de spook words (mots terrifiants), était une rumeur qui circulait dans la hacksphère depuis les années 90 . Les révélations de Snowden, et notamment le leak des slides de présentation du programme XKEYSCORE l'ont confirmé, url : <https://www.theguardian.com/ world/2013/jul/31/nsa-top-secret-program-online-data>. En témoignent aussi les consignes aux analystes du Department of Homeland Security, comme les pages 20 à 24 de ce handbook déclassifié et mis en ligne après les pressions juridiques exercées par l'Electronic Privacy Information Center sur le gouvernement américain ; url : <https://epic.org/foia/epic-v-dhs-mediamonitoring/Analyst-Desktop-Binder-REDACTED.pdf>

56. Grâce à l'ensemble des protocoles de "Traitement Automatique des Langages Naturels" (TALN) au fondement des recherches sur les logiciels de traduction par exemple, mais aussi de l'ensemble des dispositifs de manipulation du langage par l'intelligence artificielle.

57. Par son emploi du bug, elle peut être considérée comme une sous-catégorie de glitch-art selon Yves Citton, op. cit., p. 352.

58. Bret Stalbaum et al., FloodNet, interface web, The Thing, hébergé en ligne, 1990-2008, url: <https://www.thing.net/ rdom/ecd/ZapTact.html>.

59. Ibid.

60. Sur l'insurrection zapatiste comme moment de refonte des activismes numériques, voir Julian Stallabrass, Internet Art. The Online Clash of Culture and Commerce, Londres, Tate Publishing, 2003, p. 83-84.

61. On se réfère ici à Zygmunt Bauman, La vie liquide, Arles, éditions du Rouergue, 2006 ; notamment à son analyse largement partagée de la neutralisation des luttes collectives par la responsabilisation de l'individu à l'intérieur d'un réseau, et non plus d'une structure. Voir aussi l'usage critique qu'en fait Jacques Rancière, Le spectateur émancipé, Paris, La Fabrique, 2008.

62. Critical Art Ensemble, Electronic Civil Disobedience \& Other Unpopular Ideas, Automedia, 1996, p. 7.

\section{RÉSUMÉS}

La digitalisation croissante de nos existences s'est accompagnée de mutations des systèmes de contrôle, mais aussi de l'apparition de nouvelles résistances online qu'on rassemble sous l'appellation protéiforme d'hacktivismes. En faisant l'hypothèse que le web constitue un système de guidage par le langage, cet article interroge et présente les rénovations numériques des littératures politiques radicales, en montrant notamment qu'elles peuvent rêver une textualitéaction-directe au croisement des langages naturels et des langages formels. L'article s'essaye à circonscrire certaines conditions de possibilité de ces écritures hacktivistes lorsqu'elle cherchent à devenir une praxis à l'intérieur du berceau conceptuel du hack : le web. En trois temps rénovation des écritures d'agit-prop ; dénonciation des architextes autoritaires; hack des architextes autoritaires - nous revenons sur certains aspects des luttes du cyberespace, en théorie comme en pratique, tout en abordant différents exemples qui en exploitent les possibilités et répondent à des questions inattendues : qu'est-ce qu'un générateur algorithmique 
de propagande ? Le texte de Farenheit 451 peut-il brouiller les protocoles de surveillance de la NSA ? Peut-on pirater les pages web des banques pour les remplacer par de la poésie?

INDEX

Mots-clés : littérature numérique, théorie des médias, hack, hacktivisme, anarchisme

\section{AUTEUR}

\section{AURÉLIEN MAIGNANT}

FNS-Université de Lausanne - Paris 3 Sorbonne Nouvelle 